\title{
Certezas y encrucijadas del uso de I nternet en la salud pública
}

\author{
Crossroads and certainties of the use of the I nternet in the \\ public health
}

\section{Dr. C. Nancy Sánchez Tarragó}

Dirección Nacional de Epidemiología. Ministerio de Salud Pública. La Habana, Cuba.

\begin{abstract}
RESUMEN
Internet y otras tecnologías de la información tienen una presencia creciente en numerosos campos de la vida social y económica contemporánea, incluyendo los procesos y actividades relacionados con la salud. La salud pública, enfrascada en la prevención y control de las enfermedades que afectan a las poblaciones humanas, se sirve cada vez más de aplicaciones informáticas que utilizan tecnologías basadas en Internet para vigilar y predecir el comportamiento de enfermedades, analizar y visualizar datos de morbilidad y mortalidad, diseñar intervenciones y promoción de salud, así como planificar servicios y recursos. Sin embargo, el aprovechamiento de estas tecnologías se ve lastrado por inequidades socioeconómicas y retos éticos, políticos y sociotécnicos que será necesario superar. Este artículo revisa brevemente algunas certezas y encrucijadas en la utilización de los servicios y aplicaciones de Internet en la salud pública.
\end{abstract}

Palabras clave: Internet, aplicaciones web, salud pública.

\begin{abstract}
Internet and other information technologies are ever increasingly present in numerous fields of the contemporary social and economic life, including health-related processes and activities. The public health is focused on the prevention and control of the diseases that affect the human populations and it uses more and more informatic applications based on Internet for health surveillance, prediction of behavior of
\end{abstract}


diseases, analysis and visualization of morbidity and mortality data, design of health interventions and promotion, as well as for planning of services and resources. However, there are ethical, political, socio-economic and socio-technical challenges that will be necessary to overcome in order to take advantage of those web-based applications. This article briefly reviewed the crossroads and certainties of the use of Internet in the field of public health.

Key words: Internet, web-based applications, public health.

\section{NTRODUCCI ÓN}

El uso intensivo de Internet y otras tecnologías de la información y comunicación (TIC) en el campo de la salud apoya los procesos encaminados al mejoramiento de la calidad en la atención médica, al aumento del empoderamiento de usuarios y pacientes mediante un mejor acceso a la información en salud y propicia cada vez mayores posibilidades de comunicación e interacción entre los pacientes, los profesionales y las instituciones de salud, lo que permite el establecimiento de alianzas y decisiones compartidas. ${ }^{1}$

Numerosas aplicaciones que utilizan tecnologías de Internet, como las plataformas web, permiten realizar discusiones diagnósticas y presentaciones de casos clínicos entre colegas de diferentes partes del mundo, vigilar el comportamiento de enfermedades transmisibles y epidemias a través del monitoreo automatizado de grandes volúmenes de información formal e informal, mejorar el análisis, procesamiento y visualización de información en salud pública y acceder a volúmenes cada vez mayores de información científica relacionada con todos los ámbitos de las Ciencias de la Salud y la Biomedicina. Por otra parte, favorecen la participación de especialistas y público en general en el intercambio de información a través de listas de distribución, foros virtuales y blogs sobre temas de salud pública, así como la capacitación de los profesionales de la salud a través de entornos virtuales de enseñanza aprendizaje.

Sin embargo, el uso creciente de Internet también implica retos de carácter ético relacionados con estas nuevas formas de interacción entre pacientes y profesionales médicos, cuestiones de privacidad de datos, consentimiento informado, calidad de la información y equidad de acceso, tanto a las tecnologías como a la información.

Los servicios de Internet se han venido utilizando cada vez más en actividades propias de la salud pública, como la prevención y control de enfermedades, la vigilancia en salud y la promoción de conductas, comunidades y ambientes saludables. Este artículo se propone exponer algunos ejemplos de la utilización de los servicios de Internet en el campo de la Salud Pública, así como los retos y encrucijadas que conlleva su aprovechamiento.

\section{LAS CERTEZAS DEL USO DE I NTERNET EN LA SALUD PÚBLI CA}

Internet, como plataforma de servicios de información y comunicación, se ha ido expandiendo cada vez más a los diferentes campos relacionados con la salud pública. Actualmente una gran parte de los ministerios y otras instituciones de salud 
proporcionan acceso público a estadísticas de salud, como los indicadores de morbilidad y mortalidad, de recursos, servicios, entre otros. Esto no solo favorece los procesos de administración y planeación de salud, sino que también contribuye a una mayor transparencia en la gestión sanitaria de los gobiernos y a una mayor participación ciudadana en los procesos del cuidado de su salud.

Con vistas a favorecer la planeación de servicios y recursos de salud se han desarrollado aplicaciones que combinan bases de datos de morbilidad y mortalidad con sistemas de información geográfica en web (GeoWeb) que permiten visualizar a través de mapas virtuales, datos de salud pública necesarios para la planeación de servicios, y la localización y ubicación de recursos. Los sistemas de Geoweb arquetipos son Google Map y el globo virtual Google Earth. Entre los ejemplos recientes de aplicación de los GeoWeb se encuentran la representación de la ubicación y relación espacial de casos de dengue en México a través de mapas proporcionados por Google Earth, ${ }^{2}$ el monitoreo de la incidencia de la poliomielitis en las riveras de río Congo, ${ }^{3}$ el sistema de vigilancia de lesiones en Sudáfrica ${ }^{4}$ y el desarrollo del Community Health Information System en Estados Unidos. ${ }^{5}$ Estos sistemas permiten visualizar las áreas de mayor riesgo y estratificar acciones de vigilancia y control, reubicar recursos y diseñar intervenciones dirigidas a poblaciones locales.

Uno de los componentes de los sistemas de vigilancia para la salud pública es la vigilancia basada en eventos, definida como la captura organizada y rápida de información sobre eventos que presentan un riesgo potencial para la salud pública. La información puede surgir de rumores y otros informes ad hoc transmitidos tanto por conductos formales (es decir, sistemas de notificación establecidos) como informales (tales como medios de comunicación, trabajadores sanitarios e informes de organizaciones no gubernamentales). Internet es una fuente valiosa de información para la vigilancia basada en eventos, ya que existen en la web una miríada de servicios como listas de discusión, blogs, redes de reportes de enfermedades y agregadores de noticias, que contienen información sobre casos y brotes de enfermedades infecciosas. Aunque estos datos pudieran contener errores y sesgos, su actualidad y enfoque local los convierten en valiosos recursos para iniciar estudios epidemiológicos y evaluaciones de riesgo que complementan la información de otras fuentes tradicionales.

En los últimos años se han diseñado varios sistemas en plataforma web que utilizan estas fuentes informales para contribuir a la vigilancia basada en eventos. Uno de los más conocidos es el Programa para el Monitoreo de Enfermedades Emergentes ProMED mail* que comenzó siendo una lista de distribución que recopilaba información sobre enfermedades transmisibles (en humanos, animales y plantas) proveniente de diferentes fuentes tanto formales como informales, acompañada por comentarios y análisis de expertos en las diferentes temáticas que se abordaban. Actualmente ProMED mail funciona en una plataforma web, en varios idiomas, actualizada diariamente. ${ }^{6}$ Complementa la información con los servicios de otro sistema denominado Health $\mathrm{Map}^{\dagger}$. Este ha sido definido por sus fundadores como una plataforma de vigilancia en tiempo real, que permite compilar datos sobre brotes provenientes de diferentes fuentes disponibles en la web, organizados de acuerdo con la geografía, la fecha y el agente infeccioso. ${ }^{7}$ Otros ejemplos de esta nueva generación de sistemas de vigilancia que hacen minería de medios de prensa, casi en tiempo real, para reportar brotes de enfermedades infecciosas, incluyen GPHIN, desarrollado por la Agencia de Salud Pública de Canadá, ${ }^{8}$ MedISys, desarrollado por la Dirección de Salud y Asuntos de Consumidores de la Comisión Europea ${ }^{9}$ y EpiSPIDER. ${ }^{10}$

Entre las herramientas más populares de la denominada web social está Twitter, que permite colocar en la web minimensajes sobre diferentes temas y suscribirse a los que sean de interés del usuario para su seguimiento instantáneo. Una investigación 
realizada por Chunara y otros, ${ }^{11}$ analiza el volumen de noticias de prensa y de mensajes sobre cólera en Twitter durante los primeros 100 días del brote en Haití y comprueba que este volumen se corresponde con el incremento o decremento de la cantidad de casos que se reportaban oficialmente. Los investigadores concluyen que los medios informales, incluyendo Twitter, podían usarse para monitorear la actividad de los brotes de enfermedades transmisibles teniendo en cuenta además, que estos medios informales son relativamente baratos, rápidos y pueden usarse para llegar a poblaciones que de otra manera no hubieran buscado atención médica o no tendrían acceso a ella.

Otro ejemplo de la aplicación de Internet a la vigilancia en salud es el uso de la minería de millones de estrategias de búsquedas de los usuarios en motores de búsqueda como Google o Yahoo para predecir brotes o comportamientos inusuales de influenza y dengue. Estos trabajos se basan en la idea de que los individuos cuando enferman tienden a buscar información en Internet sobre su enfermedad antes de acudir a los servicios de salud o incluso, en lugar de acudir a ellos. Ginsberg, Chan y otros, ${ }^{12,13}$ mostraron que el uso de estas técnicas de minería de datos, permite estimar la actividad de las enfermedades, incluso con una antelación de una a dos semanas con respecto a los datos oficiales de vigilancia. Los productos de estas investigaciones son Google Flu Trends ${ }^{\ddagger}$ y Google Dengue Trends ${ }^{\S}$. Los autores recomiendan su utilización como complemento de los sistemas tradicionales de vigilancia.

Otra aplicación la reseña Chunara, en un artículo reciente, ${ }^{14}$ donde se describe el uso de la herramienta en plataforma web Amazon's Mechanical Turk para solicitar información sobre diagnósticos de malaria a los pobladores de varias ciudades indias. Este sitio web se basa en un concepto emergente denominado crowd sourcing, consistente en comisionar tareas a un grupo de personas dispersas geográficamente y usualmente anónimas. En este caso se pidió a los suscritos al sitio que residían en determinadas ciudades indias que autorreportaran síntomas y diagnósticos recientes de malaria. De esta manera se pudo estimar la actividad de esta enfermedad antes de las notificaciones oficiales. El artículo destaca, además, que los autorreportes se facilitaron por la amplia utilización de teléfonos móviles con conexión a Internet, así como el potencial de este método para eliminar las demoras causadas por la cadena de comandos en los que se basa la estructura tradicional de la vigilancia en salud pública.

Por otra parte, la necesidad de conocer la real incidencia de las enfermedades en viajeros internacionales, para tomar medidas de prevención, ha propiciado el desarrollo de redes internacionales colaborativas como Geosentinel" y EuroTravNet ${ }^{\mathrm{e}^{* *}}$. GeoSentinel es una red global, distribuida en los seis continentes, que recopila información sobre un amplio rango de enfermedades. Estas redes utilizan la plataforma web para el intercambio de información entre las clínicas que diagnostican a los viajeros internacionales y una base de datos centralizada. ${ }^{15}$ Un análisis de Freedman y otros, ${ }^{16}$ de la base de datos GeoSentinel permitió obtener datos sobre la proporción de la morbilidad por enfermedades transmisibles adquiridas de acuerdo con los destinos de los viajeros. En el estudio se incluyeron alrededor de 17000 viajeros con exposición en 230 áreas que fueron diagnosticados en clínicas miembros de la red en 13 países. Al examinar la morbilidad, tanto por síndromes como por diagnósticos específicos que fueron estratificados de acuerdo con la región de viajes, fue posible desarrollar un perfil completo de las infecciones que afectan a los viajeros internacionales.

En el campo de la promoción de salud y de los programas de prevención de determinantes de salud (fundamentalmente los asociados a comportamientos y estilos de vida), Internet también se ha convertido en un medio muy utilizado tanto por sus posibilidades para la amplia diseminación de los mensajes como de personalización de 
estos. ${ }^{17-19}$ La literatura sobre el tema recoge múltiples ejemplos con éxito variable de programas de intervención en Internet dirigidos a la actividad física, nutrición, cesación tabáquica y consumo de alcohol. ${ }^{20-23}$

En Cuba, la utilización de las tecnologías de la información ha acompañado al Sistema Nacional de Salud desde sus inicios, en correspondencia con el desarrollo tecnológico del momento y los recursos de los cuales ha dispuesto el país. A partir del 2001 se realizaron importantes cambios en las estrategias de informatización y se comenzó a trabajar en proyectos basados en tecnologías de Internet (XML, PHP, Web Services), software libre y otros que garantizan una explotación integrada y compatible, que se han ido introduciendo mediante la red Infomed. ${ }^{24}$

Actualmente se desarrollan varios proyectos entre el Ministerio de Salud Pública e instituciones del Ministerio de la Informática y Comunicaciones, dedicados a la gestión de información para la administración de salud y la vigilancia epidemiológica utilizando las potencialidades de las tecnologías de Internet. Entre estos proyectos cabe señalar la plataforma web SISALUD, compuesta por varios módulos que permitirían desde el Registro Informatizado de Salud (enfermedades de declaración obligatoria, registros de servicios, recursos, entre otros) hasta la gestión de sistemas específicos de administración de salud (medicamentos, logística) o de programas tales como el de vigilancia de viajeros para el Control Sanitario Internacional. ${ }^{25,26}$

\section{ENCRUCIJ ADAS DEL USO DE I NTERNET EN LA SALUD PÚBLICA}

Sin embargo, el aprovechamiento óptimo de las tecnologías de Internet se encuentra abocado a un conjunto de encrucijadas relacionadas con las inequidades de acceso tanto a las TIC como a la propia información en Internet, su calidad y confiabilidad, las competencias y habilidades de los usuarios, entre otras.

Según los datos del 2011 de la Unión Internacional de Telecomunicaciones (ITU, siglas en inglés), actualmente un tercio de la población mundial tiene acceso a Internet ( $35 \%$ de 7 mil millones). ${ }^{27}$ Según este informe, en los últimos cinco años los países subdesarrollados han aumentado su acceso a Internet de $44 \%$ en el 2006 a $62 \%$ en el 2011. Sin embargo, las disparidades entre regiones se mantienen, desfavoreciendo a los países subdesarrollados en términos de ancho de banda por usuario, acceso a Internet desde los hogares, redes de comunicación móvil de gran capacidad y precios de velocidad de conexión.

Por otra parte, la brecha digital entre naciones desarrolladas y subdesarrolladas se acompaña también de una brecha en conocimientos y habilidades para usar las tecnologías de la información, realidad socioeconómica y cultural que constituye en muchos casos una barrera importante para la utilización exitosa de las tecnologías de la información y comunicación al servicio de la salud global. ${ }^{28,29}$

Algunos estudios señalan que las propias características de los sistemas de salud pueden limitar la efectividad de la utilización de las TIC en estos proyectos, entre ellas, la complejidad de las organizaciones de salud, los altos niveles de autonomía de los principales grupos profesionales, los comportamientos tribales, las estructuras jerárquicas, los contenidos de trabajo altamente especializados y no lineales, así como la importancia de la coordinación horizontal y la colaboración entre grupos profesionales para lograr seguridad y eficiencia en el trabajo. En muchos casos, la implantación de estos proyectos, en lugar de mejorar los procesos, produce una mayor carga intelectual y operativa. Esto refuerza la necesidad de diseñar los 
proyectos utilizando enfoques sociotécnicos que introduzcan cambios profundos en los procesos y estructuras de las organizaciones de salud. ${ }^{30}$

La calidad de la información disponible en Internet también ha sido objeto de numerosos debates. En el caso de la información en salud pública, Simba llama la atención sobre el hecho de que los datos de salud recolectados en los países en desarrollo usualmente son incompletos, inexactos y carentes de oportunidad. Por tanto, no basta con introducir las TIC para mejorar la calidad de los datos, por el contrario, estas pudieran hacer más rápida la diseminación de datos incorrectos o de poca calidad que se convertirán en la "verdad" al publicarse en Internet. ${ }^{31}$

Por otra parte, la información en Internet es susceptibles de ser malinterpretada y sesgada, e incluso inducir comportamientos y prácticas contrarios a las metas de la salud pública. Estudios realizados recientemente sobre sitios web con contenidos de vacunación revelan que solo el $51 \%$ de los sitios encontrados ofrecen información correcta sobre la relación entre el autismo y la vacuna PRS (parotiditis, rubéola, sarampión), ${ }^{32}$ mientras que en otro estudio realizado en Estados Unidos en el 2009 se describe que durante una búsqueda por los términos vacunación, vacuna e inmunización, de los 30 primeros resultados que Google devolvía, 21 eran sitios sobre inmunización, de los cuales 5 de ellos podían clasificarse como sitios antinmunización. Una muestra que combinó resultados de búsqueda por el motor Canadian Google.ca, arrojó un total de 8 sitios antinmunización entre los 30 primeros resultados. ${ }^{33}$ Estudios realizados sobre este tema han demostrado que la información consultada en Internet - sea correcta o incorrecta- tiene un impacto importante en la percepción de riesgo de los usuarios y por tanto, en sus decisiones de vacunación. ${ }^{34,35}$

Otro aspecto que debe tenerse en cuenta es que la transmisión de datos a través de redes nacionales e internacionales ofrece, como ya se ha mostrado, oportunidades sin precedentes para el intercambio de información, la colaboración, el acceso y la diseminación, entre otras, pero impone nuevos retos a la confidencialidad, seguridad y privacidad de los datos. Esto obliga a la implementación de políticas de prevención y protección relacionadas con la protección física, la integridad de los datos, el acceso autorizado a la información, la preservación digital, la protección contra la divulgación no autorizada, entre otras.

La implantación de proyectos basados en tecnologías de Internet en Cuba no escapa de esta dualidad entre el aprovechamiento de tecnologías que han demostrado ser exitosas en determinados campos de la salud pública y las propias complejidades de utilización.

Aunque Cuba se encuentra en el $5 \%$ de penetración de Internet desde los hogares, según datos de la ITU, ${ }^{36}$ su uso social a través de sitios públicos en universidades, J oven Club de Computación, instituciones de investigación y otros, ha propiciado su amplio aprovechamiento. De hecho, el propio informe de la ITU antes citado clasifica a Cuba en el número uno de los países de la región de Las Américas en el subíndice aptitud hacia las TIC, o sea, capacidad para usarlas con eficacia debido a este uso social.

En el Sistema Nacional de Salud existen 603 bibliotecas médicas de hospitales, institutos de investigación, centros de enseñanza medica superior y policlínicos, así como 807 instituciones médicas en los tres niveles de atención médica conectadas a la red Infomed, ${ }^{37}$ lo que potencialmente les permitiría aprovechar las ventajas de la Biblioteca Virtual de Salud, puerta de acceso a un gran volumen de información científica nacional e internacional, así como a los proyectos de informatización que actualmente desarrolla el Ministerio de Salud Pública. Muchas de estas instituciones, 
aunque en menor cuantía, también cuentan con acceso pleno a los servicios web en Internet.

Sin embargo, la ampliación de los servicios de Internet en el Sistema Nacional de Salud está signada por complejos factores económicos y políticos que conforman el contexto de penetración de Internet en el país en relación con el ancho de banda, velocidad de conexión y disponibilidad de computadoras. Por otra parte, la utilización de los servicios ya disponibles también depende de la cultura organizacional y las competencias informacionales y tecnológicas de sus miembros. En el año 2007 se realizó una exploración del uso de servicios de web social por parte de investigadores de institutos nacionales de investigación en Cuba, que puso de manifiesto la falta de familiarización de estos con los blogs, wikis y canales RSS***. ${ }^{38}$ Evidentemente, esta situación es parte de la brecha digital y de conocimientos a la que se hacía referencia en párrafos anteriores y que afecta fundamentalmente a nuestros países subdesarrollados.

\section{CONSI DERACI ONES FI NALES}

La revisión de la literatura que respalda este trabajo permite apreciar las potencialidades de Internet, no solo como fuente de información científico-técnica, sino también como plataforma de servicios y aplicaciones que aumentan las posibilidades de análisis, procesamiento y visualización de datos útiles para tomar decisiones en la prevención y control de enfermedades, así como herramientas que favorecen intervenciones de salud en determinadas poblaciones, colaboraciones institucionales y la participación activa de los propios ciudadanos en la notificación de enfermedades y en el cuidado de su salud.

No obstante, también se han puesto de manifiesto los obstáculos de variada índole que pueden afectar su aprovechamiento y efectividad. Particularmente en Cuba, la implantación y utilización de proyectos de informatización están signadas por dificultades económicas y barreras externas que se traducen en pobre ancho de banda y velocidad de conexión e insuficientes computadoras. Sin embargo, estas también pudieran estar afectadas por una cultura organizacional que en ocasiones no propicia comportamientos creativos y participativos con las TIC, así como por insuficientes competencias informacionales y tecnológicas que hagan a los individuos protagonistas de transformaciones en el ámbito de la salud pública con el apoyo de estas herramientas.

\section{REFERENCI AS BI BLI OGRÁFICAS}

1. Eysenbach G. What is e-health? J Med Internet Res. 2001;3(2):e20.

2. Lozano-Fuentes S, Elizondo-Quiroga D, Farfan-Ale JA, Loroño-Pino MA, GarciaRejon J, Gomez-Carro S, et al. Use of Google Earth ${ }^{\mathrm{TM}}$ to strengthen public health capacity and facilitate management of vector-borne diseases in resource-poor environments. Bull World Health Org. 2008; 86(9): 718-25.0

3. Kamadjeu R. Tracking the polio virus down the Congo River: a case study on the use of Google Earth (TM) in public health planning and mapping. Int J Health Geogr [Internet]. 2009 [cited 2012 May 20]; 8:4. Available from: http://www.ijhealthgeographics.com/content/8/1/4

http://scielo.sld.cu 
4. Cinnamon J, Schuurman N. Injury surveillance in low resource settings using Geospatial and Social Web technologies. Int J Health Geogr [Internet]. 2010 [cited 2012 May 20]; 9:25. Available from: http://www.ijhealthgeographics.com/content/9/1/25

5. Highfield L, Arthasarnprasit J, Ottenweller CA, Dasprez A. Interactive web-based mapping: bridging technology and data for health. Int J Health Geogr [Internet]. 2011 [cited 2012 May 20]; 10: 69. Available from: http://www.ij-

healthgeographics.com/content/10/1/69

6. Woodall J, Calisher CH. ProMED-mail: Background and purpose. Emerg Infect Dis. $2001 ; 7: 563$.

7. Brownstein JS, Freifeld CC, Reis BY, Mandl KD. Surveillance sans frontières: Internet-based emerging infectious disease intelligence and the HealthMap project. PLoS Med. 2008; 5(7): e151.

8. Mykhalovskiy E, Weir L. The Global Public Health Intelligence Network and early warning outbreak detection: A Canadian contribution to global public health. Can J Public Health. 2006; 97:42-4.

9. Health Threats Unit at Directorate General Health and Consumer Affairs of the European Commission. MedI Sys (Medical Intelligence System) [Internet]. 2007 [cited 2012 May 20]. Available from: http://medusa.jrc.it/medisys/homeedition/es/home.html

10. Tolentino H, Kamadjeu R, Fontelo P, Liu F, Matters M. Scanning the emerging infectious diseases horizonVisualizing ProMED emails using EpiSPIDER. Adv Dis Surveill. 2007;2: 169 .

11. Chunara R, Andrews JR, Brownstein JS. Social and news media enable estimation of epidemiological patterns early in the 2010 Haitian cholera outbreak. Am J Trop Med Hyg. 2012; 86: 3645.

12. Ginsberg J, Mohebbi MH, Patel RS, Brammer L, Smolinski MS, Brilliant L. Detecting influenza epidemics using search engine query data. Nature. 2009; 457: 1012-4.

13. Chan EH, Sahai V, Conrad C, Brownstein JS. Using Web Search Query Data to Monitor Dengue Epidemics: A New Model for Neglected Tropical Disease Surveillance. PLoS Negl Trop Dis. 2011;5(5): e1206. doi: 10.1371/journal.pntd.0001206.

14. Chunara R, Chhaya V, Bane S, Mekaru SR, Chan EH, Freifeld CC, et al. Online reporting for malaria surveillance using micro-monetary incentives, in urban India 2010-2011. Malaria J. 2012; 11: 43.

15. Torresi J, Leder K. Defining infections in international travellers through the GeoSentinel surveillance network. Nature Reviews Microbiol. 2009; 7:895-901. doi: $10.1038 /$ nrmicro2238.

16. Schneider F, van Osch L, de Vries H. I dentifying Factors for Optimal Development of Health-Related Websites: A Delphi Study Among Experts and Potential Future Users. J Med Internet Res. 2012; 14(2): e43. doi: 10.2196/jmir.1788 
17. Bennett GG, Glasgow RE. The Delivery of Public Health Interventions via the Internet: Actualizing Their Potential. Ann Rev Public Health. 2009; 30: 27392.

18. Robroek SJ W, Lindeboom DEM, Burdorf A. I nitial and Sustained Participation in an Internet-delivered Long-term Worksite Health Promotion Program on Physical Activity and Nutrition. J Med Internet Res [Internet]. 2012 [cited 2012 May 20]; 14(2): e43. Available from: http://www.jmir.org/2012/2/e43/

19. Krebs P, Prochaska JO, Rossi JS. A meta-analysis of computer-tailored interventions for health behavior change. Prev Med. 2010;51(3-4):214-21.

20. van den Berg MH, Schoones JW, Vliet Vlieland TP. Internet-based physical activity interventions: a systematic review of the literature. J Med Internet Res.

$2007 ; 9(3): \mathrm{e} 26$.

21. Neville LM, O'Hara B, Milat AJ. Computer-tailored dietary behaviour change interventions: a systematic review. Health Educ Res. 2009;24(4):699-720.

22. Myung SK, McDonnell DD, Kazinets G, Seo HG, Moskowitz J M. Effects of Web- and computer-based smoking cessation programs: meta-analysis of randomized controlled trials. Arch Intern Med. 2009; 169(10): 929-37.

23. White A, Kavanagh D, Stallman H, Klein B, Kay-Lambkin F, Proudfoot J, et al. Online alcohol interventions: a systematic review. J Med Internet Res.

2010; 12(5): e62.

24. Delgado Ramos A, Vidal ledo M. La Informática en la salud pública cubana. Rev Cubana Salud Pública [Internet]. 2006[citado 20 May 2012];32(3). Disponible en: http://scielo.sld.cu/scielo.php?script=sci_arttext\&pid=S086434662006000300015\&lng=es

25. Cabrera Hernández M, Delgado Ramos A, Sánchez Rodríguez A, Acuña Sánchez A, Barrios A, Hernández Laborde A, et al. Plataforma para la administración, procesamiento y transmisión de la información en el Sistema de Salud (SISALUD). VI Congreso Internacional de Informática en la Salud; 9 al 13 de febrero 2011[Internet]. La Habana: VI Congreso Internacional; 2011 [citado 20 May 2012]. Disponible en: http://www. informatica2009.sld.cu/Members/mirnacabrera/plataforma-para-laadministracion-procesamiento-y -transmision-de-la-informacion-en-el-sistema-desalud-sisalud /?searchterm=sisalud

26. Gonzalez Tolmo D, Fernandez Marin MA, Valdez Diaz A. Sistema para el Control Sanitario Internacional. VII Congreso Internacional de Informática en la Salud; 7 al 11 de febrero 2009 [Internet]. La Habana: VII Congreso Internacional; 2009 [citado 20 May 2012]. Disponible en:

http://www.informaticasalud2011.sld.cu/index.php/informaticasalud/2011/paper /view/109

27. ITU Telecom World. ICT Facts and figures 2011[Internet]. 2012 [citado 20 May 2012]. Disponible en: http://www.itu.int/ITU-D/ict/

28. Missena C, Cook TM. Appropriate information-communications technologies for developing countries. Bull World Health Org. 2007; 85(4):248. doi: 10.2471/BLT.07.041475. 
29. Simba DO. Application of ICT in strengthening health information systems in developing countries in the wake of globalisation. African Health Sciences.

2004; 4(3): 195-9.

30. Rodrigues RJ, Risk A. Health in Latin America and the Caribbean: Development and Policy Issues. J Med Internet Res [Internet]. 2003 [cited 2012 May 20 ]; 5(1): e4. Available from: http://www.jmir.org/2003/1/e4/

31. Westbrook J, Braithwaite J, Gibson K, Paoloni R, Callen R, Georgiou A et al. Use of information and communication technologies to support effective work practice innovation in the health sector: a multi-site study. BMC Health Services Research. 2009; 9: 201. doi: 10.1186/1472-6963-9-201

32. Kata A. A postmodern Pandora's box: Anti-vaccination misinformation on the Internet. Vaccine. 2010;28(7): 170916.

33. Scullard P, Peacock C, Davies P. Googling children's health: reliability of medical advice on the internet. Arch Dis Child. 2010;95(8):580-2.

34. Betsch C, Renkewitz F, Betsch T, Ulshöfer $C$. The influence of vaccine-critical websites on perceiving vaccination risks. J Health Psychol. 2010; 15(3):446-55.

35. Betsch C. Innovations in communication: the Internet and the psychology of vaccination decisions. Euro Surveill. 2011[cited 2012 May 20]; 16(17): pii=19852. Available from: http://www. eurosurveillance.org/ViewArticle.aspx?Articlel d=19852

36. ITU Perfiles Estadísticos de la Sociedad de la Información 2009 [Internet]. Región de América: Sociedad; 2009 [citado 20 May 2012]. Disponible en:

http://www.itu.int/en/Pages/default.aspx

37. República de Cuba. Anuario Estadístico de Salud 2011[Internet]. La Habana: Ministerio de Salud Pública;2012 [citado 20 May 2012]. Disponible en: http://files.sld.cu/dne/files/2012/04/anuario-2011-e.pdf

38. Sánchez Tarragó N, Fernández-Molina JC. Journal reading and publishing patterns of Cuban health researchers. Information Development [Internet]. 2009 [citado 20 May 2012];25(4):283-95. Disponible en: http://intlidv. sagepub. com/content/25/4/283. full. pdf+html

* Disponible en: http://www.promedmail.org

† Disponible en: http://www.healthmap.org

‡ Disponible en: http://www.google.org/flutrends

$\S$ Disponible en: http://www.googlr.org/denguetrends

ๆ Disponible en: http://geosentinel.org

** Disponible en: http://www.trop.net

http://scielo.sld.cu 
*** Los canales RSS permiten a un sitio Web, principalmente a aquellos que tienen actualizaciones frecuentes, informar a los interesados sobre las novedades del sitio, típicamente por medio de la distribución de titulares de noticias y contenidos breves. Cuando el usuario observa un elemento de interés en su canal, puede seguir el enlace para obtener el contenido completo. (Sánchez Tarragó N. Sindicación de contenidos con canales RSS: aplicaciones actuales y tendencias. ACI MED. 2007;15(3). Disponible en: http://scielo.sld.cu/scielo. php?script=sci_arttext\&pid=S1024-

$\underline{94352007000300003 \& \operatorname{lng}=\mathrm{es}}$

Nancy Sánchez Tarragó. Dirección Nacional de Epidemiología. Ministerio de Salud Pública. Calle 23 esq. N. El Vedado 10400. La Habana, Cuba.

Correo electrónico: sanchezn@infomed.sld.cu 